\title{
Sérotonine ou la quête du bonheur selon Michel Houellebecq
}

Ruth Amar, Université de Haïfa

Porter témoignage d'un monde qui risque de basculer à tout moment, telle semble être la finalité des romans de Houellebecq, qui reflètent les caractéristiques essentielles de la société contemporaine. Sandrine Rabosseau évoque le fait que «Houellebecq adopte systématiquement un discours sociologique dans son œuvre romanesque »(43-44). Comme Emile Zola, il aborde « en toute liberté les sujets explosifs de la vie occidentale : le travail, l'argent, la sexualité, la famille » (44). David Jérôme, quant à lui, affirme que Michel Houellebecq est :

au milieu du monde. Il ne se tient pas comme on pourrait penser tout d'abord, à côté ou à l'écart du monde. Ce n'est pas non plus une position de marginal. Mais le milieu n'est pas non plus le centre. Il n'est pas non plus « au centre du monde » comme une subjectivité abstraite qui légifère et essaie de soumettre le monde à ses lois subjectives (pseudo-loi de l'intime). « Au milieu du monde » signifie se tenir dans le monde comme un élément, une particule élémentaire en suspension. L'observation peut commencer dès lors que le monde est saisi comme milieu et, avec elle, le compte-rendu descriptif. (142)

Dans ce « compte-rendu descriptif », l'idée du bonheur n'est pas entièrement bannie de la vie des personnages et, ici et là, elle les effleure. Le narrateur houellebecquien « avoue sa disgrâce : il la hurle à chacune [des pages]. Lire MH sans tout comprendre de travers, c'est voir que ses romans sont une longue plainte sur le naufrage d'une civilisation...»(Viard 10). Cette plainte naîtrait effectivement d'un besoin essentiel d'être heureux. Dans les textes apparaissent des échappées d'espoir sur le fond de l'accablement quotidien éprouvé par les personnages. Houellebecq explique : «Notre malheur n'atteint son plus haut point que lorsqu'a été envisagée, suffisamment proche, la possibilité du bonheur »(Interventions, 245). Même si la quête s'avère difficile et frustrante, et même si un sentiment d'échec constant s'impose dans les romans, la quête du bonheur ne cesse d'occuper une place fondamentale dans l'œuvre.

En 1997, Houellebecq écrit son recueil de poèmes La poursuite du bonheur ; le mot, l'idée ou l'image du bonheur apparaissent ici et là à travers l'œuvre entière, et souvent dans des vers inclus dans sa prose : «Et l'amour, où tout est facile, / Où tout est donné dans l'instant ; / Il existe au milieu du temps, / La possibilité d'une île » (Possibilité, 433). Dans Interventions, Houellebecq affirme : «J'aimerais bien échapper à la présence obsessionnelle du monde moderne ; rejoindre 
un univers à la Mary Poppins, où tout serait bien. Je ne sais pas si j'y parviendrai » (47). Cet univers idéal transparaît parfois entre les lignes et, occasionnellement, il est mis en scène de façon directe. Ce sont justement des moments rares de vérité profonde où, dans Les particules élémentaires, Bruno avoue à Christiane : «Je crois que je suis heureux » (276). Alors que le néohumain Daniel 24 de La possibilité d'une île annonce : « Je mène une vie calme et sans joie 》 (77), son prédécesseur Daniel 1 affirme : « cette semaine à Madrid, où je fus presque tout le temps avec Esther, $[\ldots]$ reste une des périodes les plus heureuses de ma vie [...]. Pour la première fois de ma vie je me sentais, sans restrictions, heureux d'être un homme » (221). Cette déclaration est le résultat de la relation entre Daniel1 et Esther : «Pour la première fois de ma vie je me sentais animé à l'égard d'autrui d'intentions charitables et amicales, j'aurais aimé que tout le monde soit heureux comme je l'étais moi-même » (222). Daniel 1, alors qu'il tombe amoureux, repense aux griefs de la solitude et découvre combien il est bon de partager sa vie avec une compagne : «A quoi bon maintenir en état de marche un corps qui n'est touché par personne ?» (222), ce qui lui fait dire : « immense et admirable, décidemment, était la puissance de l'amour » (222).

A Marie Lebrun qui interroge Houellebecq sur le fait que dans Rester vivant, méthode, « le monde est une souffrance déployée », il répond : «Si. C'est cela ». Mais il prend soin d'ajouter : «Avec des moments de bonheur bien sûr. Il y a une citation de Schopenhauer que je n'ai pas réussi à placer dans mon roman et qui pourtant me plaisait particulièrement, c'est que 'la possibilité du bonheur devrait subsister, à titre d'appât' » (Interview, internet). L'idée du bonheur serait une sorte d'astuce de l'existence, une tentation afin d'encourager l'être humain à continuer à vivre.

Lecteur boulimique et érudit, Houellebecq convoque dans ses romans quantité d'auteurs, qu'il cite plus ou moins littéralement. Deux d'entre eux, à savoir Auguste Comte, père du positivisme, et Arthur Schopenhauer, maître spirituel de Houellebecq, sont vraisemblablement les influences majeures de la conception du bonheur dans l'œuvre. Apparemment, ces deux philosophes n'ont rien en commun, mais il est possible de repérer certains points similaires concernant l'idée du bonheur exprimée dans les romans de Houellebecq. Dans son dernier livre Sérotonine, l'intérêt porté au bonheur se précise encore plus. Cette fois, il n'est plus question de philosophie : le titre scientifique surgit sous la forme d'une hormone, un neurotransmetteur à l'origine de notre humeur, utilisée dans les antidépresseurs pour une meilleure santé mentale. Estce à dire que Houellebecq a renoncé au bonheur ? Ou bien est-ce, au contraire, une nouvelle 
tentative de résistance ? Je tenterai dans cette brève étude de répondre à la question et voir si la quête du bonheur persiste dans son dernier roman.

Avant de nous tourner vers Sérotonine, il est pertinent de retracer le chemin de la quête du bonheur sous l'influence de Comte et de Schopenhauer. Le positivisme scientifique de Comte atteste que l'esprit scientifique, à l'aide d'une loi inexorable du progrès de l'esprit humain, appelée «loi des trois états» (théologique, métaphysique et positiviste) ${ }^{1}$, remplace les croyances théologiques ou les explications métaphysiques. Comte préconise une religion de l'humanité sans Dieu, où l'être humain doit adorer l'humanité en soi.

Les éléments de la doctrine de Comte sont à la base de La possibilité d'une île où Dieu n'existe pas et où un nouveau monde est créé à partir d'une philosophie positiviste. La nécessité d'une religion où l'Humanité est un Dieu qui se crée lui-même, ceci par la croyance et l'action de chacun de ses membres, est fondatrice de la secte des raëliens, alors que l'auteur prédit la mort des grandes religions monothéistes. En effet, le mouvement raélien prépare l'accueil sur terre des extraterrestres en diffusant leur message dans le monde. Ainsi Daniel 1 porte-t-il son refus du vieillissement à l'extrême : il refuse le dépérissement du corps pour lui préférer le suicide ou l'euthanasie. Il lèguera tous ses biens à cette secte qui justifie une nouvelle religion : les Elohimites. Plus de famille, c'est la «Sœur Suprême » qui gouverne le monde où le patronyme est remplacé par un chiffre.

En devenant «positif », l'esprit renoncerait à la question du «pourquoi ? (chercher les causes premières des choses) et se limiterait surtout au « comment », à la formulation des lois de la nature exprimées en langage mathématique, en dégageant, par le moyen d'observations, les relations constantes qui unissent les phénomènes et expliquent la réalité. Cette approche «positive » est bien ressentie dans l'atmosphère indifférente de La possibilité d'une île, où les questions de raisonnement ne se posent plus, où seuls les récits de vie des prédécesseurs servent de livre d'histoire lu et relu impassiblement par les néo-humains pacifiés.

Irrémédiablement unis au niveau le plus intérieur, bonheur et douleur se confrontent chez Comte : «Au milieu des plus graves tourments qui puissent jamais résulter de l'affection, je n’ai jamais cessé de sentir que l'essentiel pour le bonheur c'est toujours d'avoir le cœur dignement rempli, même de douleur, oui même de douleur, de la plus amère douleur » (Correspondances, 360). Cet aphorisme apparemment construit sur le paradoxe «bonheur-douleur » plane également au-dessus de l'œuvre de Houellebecq : la douleur fait partie du bonheur. Ou plutôt, c'est la douleur 
qui engendrerait le bonheur. Mais le bonheur égale-t-il les souffrances supportées pour l'obtenir ? Cette question est essentielle en ce qui concerne les textes de Houellebecq, car c'est à ce stade que la philosophie de Comte rejoint celle de Schopenhauer et où les deux doctrines s'entrecroisent dans les romans.

Schopenhauer insiste sur le fait que toute existence est souffrance, puisque l'être humain cherche incessamment à combler un manque. L'homme ne peut être heureux qu'à condition d'atteindre le « quiétisme ». Autrement dit, l'essence de l'être étant un effort douloureux (dont la libération est dans l'intelligence, l'ascétisme moral et la pitié), il faut abolir en soi le vouloir-vivre. Mais plus précisément, à propos de la douleur, Schopenhauer affirme qu'elle « possède en puissance une vertu sanctifiante » et il ajoute : «c'est ce qui explique pourquoi un grand malheur, une profonde souffrance ne va jamais sans inspirer un certain respect » (496).

Cette idée est exprimée dans l'œuvre de Houellebecq qui proclame dès les premières pages de Rester vivant que la souffrance engendre l'écriture : «Toute souffrance est utile» (9). Le narrateur de L'extension précise : «Si je n'écris pas ce que j’ai vu, je souffrirai autant - et peutêtre un peu plus. Un peu seulement, j'y insiste. L'écriture ne soulage guère. Elle retrace, elle délimite » (14). Schopenhauer accentue le fait qu'en passant par les différentes étapes de la souffrance, l'être humain peut atteindre le nirvana, stade où la volonté est entièrement abolie (influence connue du bouddhisme sur le philosophe) : «Il n'y a qu'un seul chemin qui conduise au salut; il faut que la volonté se manifeste sans obstacle, afin que dans cette manifestation elle puisse prendre connaissance de sa propre nature. Ce n'est que grâce à cette connaissance que la volonté peut se supprimer elle-même, et par le fait en finir avec la souffrance aussi » (502). Il est alors possible de concevoir le bonheur, qui serait la fin du processus de la souffrance. Cette idée est exprimée dans les textes de Houellebecq. Dans L'extension, Tisserand souffre de frustration sexuelle et de la cruelle compétition entre les hommes. Les mêmes caractéristiques se reproduisent dans Plateforme, avec le tourisme sexuel censé apporter une solution cynique aux Européens ; dans Les particules élémentaires, la liberté sexuelle est destinée à résoudre les problèmes, bien qu'en fin de compte, elle les aggrave. Bruno finit dans un asile. Si, dans les premiers romans, la souffrance était déployée, à partir des Particules, elle s'estompe au fil du texte où on entrevoit une solution à tous les malheurs de l'homme par la suppression de la reproduction sexuelle et son remplacement par le clonage (par l'intermédiaire de Michel le savant). 
Avec La possibilité d'une île, cette solution s'affermit sous formes de clones de Daniel 1. Les néo-humains vivent dans un monde qui n'est ni édénique ni misérable. Réalisation du nirvana bouddhique, c'est d'un monde homéostatique qu'il s'agit. Le système qui mène les néo-humains à conserver leur équilibre énergique, est la maintenance de l'ensemble des paramètres physicochimiques de l'organisme qui restent relativement constants et semblent ne pas changer d'un néohumain à l'autre. D'ailleurs, la fixité du milieu intérieur est la condition d'une vie libre et indépendante ; afin d'évoluer, les néo-humains ne sont aucunement préoccupés par la société ou la communication entre eux dans un univers où le principe du plaisir, aussi bien que celui du désir et de la reproduction ont été complètement anéantis. Le seul compagnon du « néo humain » est le chien, lui-même clone du chien premier de Daniel 1. Les relations entre hommes et femmes se font par écran où l'on correspond et échange des informations et parfois, de manière inattendue, des poèmes (peut-être, tout de même, pour signaler ce qui déclenchera un jour ne nouvelle mutation, cette fois culturelle, vers les sentiments tels qu'ils existaient chez les humains). Daniel 25 explique :

... la jalousie, le désir et l'appétit de procréation ont la même origine, qui est la souffrance d'être. C'est la souffrance d'être qui nous fait rechercher l'autre, comme un palliatif ; nous devons dépasser ce stade afin d'atteindre l'état où le simple fait d'être constitue par luimême une occasion permanente de joie ; où l'intermédiation n'est plus qu'un jeu, librement poursuivi, non constitutif d'être. Nous devons atteindre en un mot à la liberté d'indifférence, condition de possibilité de la sérénité parfaite. (376)

Selon Schopenhauer, la sexualité représente l'expression du vouloir vivre et, en l'abolissant, il est possible d'éviter la souffrance et d'atteindre la sérénité. Cette idée se manifeste d'abord dans Les particules élémentaires où Michel Djerzinsky se consacre à la science, qui lui procure une satisfaction de loin supérieure à la sexualité et, ensuite, dans La possibilité d'une île où Daniel 24 et Daniel 25, ignorant tout de la sexualité, sont désormais des néo-humains impassibles. Seuls ces protagonistes peuvent parvenir à la connaissance parfaite et à l'objectivité pure, évoquant celle bien connue de Schopenhauer.

La quête du bonheur présente dans l'œuvre est essentiellement reliée au sens du devoir que doit s'imposer tout homme digne de ce nom comme l'affirme Houellebecq dans Interventions : « Il n'y a que le sens du devoir qui puisse réellement nous maintenir en vie. Concrètement, si l'on souhaite se doter d'un devoir pratique, on doit faire en sorte que le bonheur d'un autre être dépende de votre existence; on peut par exemple essayer d'élever un enfant jeune, ou à défaut acheter un caniche »(41). Il est frappant de constater qu'au sein même de textes dont la noirceur est 
incontestable, la notion du devoir apparaît comme un élément porteur de sens. On y découvre que l'être humain doit accomplir des actes en vertu de sa qualité humaine, qui se doit de respecter autrui. Il apparaît donc que, finalement, l'individu a besoin d'autrui pour se réaliser. Le problème est que, dans les écrits de Houellebecq, on découvre une opposition implicite entre passions et devoir. Les passions considérées généralement comme négatives (l'étymologie du terme renvoie à la passivité - le mot vient du latin «patior», qui signifie «souffrir ») s'imposent à nous de manière involontaire. Elles sont une affection durable de la conscience dont l'origine, pour la plupart des philosophes, se trouve dans le corps. Le devoir implique le sacrifice de ses envies personnelles, des désirs égoïstes, mais permet à l'être humain de gagner le respect et, ultimement, le bonheur.

\section{Sérotonine et Captorix : la dernière chance d'être heureux ?}

Qu'advient-il de la quête du bonheur dans le dernier roman de Houellebecq ? Vingt-huit ans après La poursuite $d u$ bonheur, quatorze ans après La possibilité d'une île, il suffit de lire le titre (plus ironique tout de même, ce qui éveille en nous un doute sur le but du roman) du roman pour se rendre compte que Houellebecq n'a pas renoncé à la question. Dans un entretien récent au Danemark où on l'interroge à propos de Sérotonine et sur sa définition du bonheur, Houellebecq répond :

Royaume limité. C'est un état dans lequel on peut vivre. Un état dont on souhaite la perpétuation en soit... en fait c'est entièrement opposé à la notion d'impermanence... mais c'est plus facile à définir négativement. C'est plus facile de dire ce que le bonheur n'est pas. Ce qui est contraire au bonheur que dire ce qui est le bonheur... le passé est toujours heureux et finalement l'avenir aussi... il n'y a que le présent qui fasse mal... (Interview Humlebæk).

Est-ce à dire que le bonheur ne se dévoile qu'après coup ? Même limité, s’il était accompli, quelque chose du bonheur serait déjà perdu. De fait, le bonheur recule toujours plus loin sur la ligne de fuite idéale d'un présent désorienté, où les personnages sont finalement censés à « faire avec ». Le bonheur suppose sa reconduction à chaque instant. Il doit pouvoir se renouveler du dedans de luimême et au-delà de toute satiété.

Il faut aussi tenir compte du fait que, dans la société contemporaine, se développe la volonté d'une jouissance illimitée qui est également reflétée dans l'œuvre de Houellebecq. A la question «D'où vient cette idée du bonheur ? Est-ce une nouvelle idée fabriquée par la société de 
consommation ? Ou est-ce un souvenir de quelque chose que nous avons perdu ? $\gg$ Houellebecq répond :

C'est très français. C'est une allusion à une phrase assez célèbre en France : « Le bonheur est une idée neuve en Europe ». St Just... C'est une phrase qui m'exaspère... Ce que je veux dire c'est que le bonheur est une ancienne idée européenne. Je reconnais que la phrase de St Just est excellente... Je considère naturellement qu'il faut faire son devoir et que le bonheur est donné par surcroit éventuellement. Mon premier recueil de poèmes La poursuite du bonheur était une allusion négative à la Constitution Européenne qui place parmi les buts de la société humaine la poursuite du bonheur... Quelle absurdité ! On ne peut pas constituer une société là-dessus c'est évident. (Interview Humlebæk)

Ces propos évoquent ce que Pascal Bruckner expliquait à propos du bonheur au vingt-et-unième siècle - que la conception même du bonheur favorise ce processus dans la société moderne. Bruckner reproche à cette société le « devoir de bonheur » (Euphorie perpétuelle, 19), posture qui s'est généralisée à la fin du vingtième siècle et qui entraîne l'être moderne à tout apprécier selon la culture du plaisir et du désagrément, l'invitant à l'euphorie et au rejet dans la honte de ceux qui ne s'y adaptent pas. Les conditions psychiques du bonheur d'exister paraissent de plus en plus plausibles. Selon Gérard Pommier, nous sommes « devant une tâche nouvelle dans l'histoire de l'humanité : le bonheur est à portée de main. Et l'on se demande avec curiosité si cette possibilité elle-même ne va pas devenir son principal obstacle » (100).

Les conséquences conduisent au malaise qui s'empare d'un nombre toujours croissant de sujets et qui dégénère en un désespoir envahissant, un sentiment de vide intérieur et d'absurdité de la vie, caractéristiques saisies dans les textes de Houellebecq. Le phénomène d'individuation auquel l'homme moderne est sujet ne lui permet qu'un bonheur superficiel' ${ }^{2}$.

Sérotonine, qui garde l'atmosphère sombre et lugubre des romans précédents avec les mêmes thèmes fondateurs, met en scène un héros narrateur, Florent-Claude Labrouste, quarantesix ans, « qui [n'a] jamais été capable de contrôler [sa] propre vie » (12), qui est accablé par le contact social, nihiliste, opprimé par un univers qu'il considère absurde et vide de sens. FlorentClaude est ancien ingénieur agronome et, comme les autres protagonistes de l'œuvre, c'est un personnage qui endure la solitude, la décadence, le sexe dépourvu d'amour. Souffrant de manque permanent, il fume : «le soulagement que m'apporte la première bouffée est immédiat, d'une violence stupéfiante. La nicotine est une drogue parfaite, une drogue simple et dure, qui n'apporte aucune joie, qui se définit entièrement par le manque, et par la cessation du manque » (9). FlorentClaude décide de se soustraire à la vie sociale : il disparaît en s'installant dans un hôtel (l'un des 
rares qui hébergent les fumeurs) après avoir quitté Yuzu, sa compagne, une Japonaise de vingt-six ans qui copule, entre autres, avec des chiens, ce qui l'écœure «mais surtout pour les chiens » (54).

$\mathrm{Si}$, dans son recueil de poésie La poursuite du bonheur et ses autres textes, Houellebecq évoquait le bonheur de manière thématique et philosophique comme nous venons de le voir, dans ce dernier roman c'est sous la forme d'«un petit comprimé blanc, ovale, sécable [...] d'une efficacité surprenante» (12) qu'il apparaît : le captorix - un antidépresseur - que le narrateur dépressif se fait prescrire par un médecin (comme des millions d'Européens). Le captorix permet aux patients « d'intégrer avec une aisance nouvelle les rites majeurs d'une vie normale au sein d'une société évoluée » en « favorisant la libération par exocytose de la sérotonine » (12). Il permet une vie calme mais sans enthousiasme ou joie excessive pour, finalement, supporter l'absurdité de l'existence sans souffrir, ce qui évoque encore une fois la solution qui se manifestait déjà dans $L a$ possibilité d'une île. Cette fois, ce qui remplace le mécanisme des clones c'est le petit comprimé, ce qui dote le roman d'un réalisme flagrant. Mais le bonheur se définit-il par l'absence de souffrance ? En tout cas, dans Sérotonine, le narrateur se pose la question de manière directe dès qu'il emménage dans la petite chambre d'hôtel: «Etais-je capable d'être heureux dans la solitude ? Je ne le pensais pas. Etais-je capable d'être heureux en général ? C'est le genre de questions, je crois, qu'il vaut mieux éviter de se poser » (88).

Camille, son seul véritable amour, la femme avec qui Florent-Claude a partagé sa vie pendant cinq ans, l'a quitté quand elle a découvert qu'il la trompait avec Tam au «joli petit cul de black ». Après leur rupture, elle s'installe comme vétérinaire en Normandie, où elle vit seule avec un enfant de cinq ans, fruit d'une rencontre téméraire. Florent-Claude espère la reconquérir et, pour y parvenir, va jusqu'à vouloir assassiner son enfant, ce qui concède à tout le récit un côté excentrique et fantastique évoquant l'incitation au meurtre du «jeune Noir» (116) dans L'extension. Les assassinats respectifs sont prémédités, mais finalement entravés. Dans Sérotonine, le meurtre prévu mais avorté pourrait s'interpréter comme une métaphore de la fin de la civilisation occidentale incapable de procréer, ou comme l'image de la femme inaccessible dès qu'elle est mère. Cette scène peut aussi être qualifiée de clin d'œil au polar, mais bien sûr, un polar raté où le suspens n'aboutit à rien tout comme la vie de Florent-Claude et le récit lui-même.

L'ami du narrateur, Aymeric, victime de la politique libérale de l'Union Européenne et plaqué par une femme refusant de s'enterrer dans un village paumé, se noie dans l'alcool. Il tente d'améliorer son sort et celui de ses collègues. Le constat est irrémédiable : le capitalisme libéral a 
créé de faux besoins et ne rend pas les gens heureux. La mondialisation a tué le monde paysan. Tenter d'agir individuellement est quasiment impossible, puisque tout se décide au-dessus et ailleurs. Florent-Claude, qui comprend qu'il est un agronome sans aucun pouvoir, déclare avec amertume : «...qui étais-je pour avoir cru que je pouvais changer quelque chose au mouvement du monde ?» (251). Ceci renforce l'idée que la société contemporaine est conçue autrement. Tout se passe comme si les individus n'ont plus aucun contrôle de leur propre existence. La mondialisation et la technologie, le cyber et l'internet décident pour les êtres humains ce qui est bon ou mauvais. Il reste peu de place pour la vie privée ou les sentiments. Le bonheur est tout simplement impossible : «Plus personne ne sera heureux en Occident, plus jamais » (102), affirme Claire, l'une des partenaires de Florent-Claude, « nous devons considérer le bonheur comme une rêverie ancienne, les conditions historiques n'en sont tout simplement plus réunies » (102). Florent-Claude en évoquant son amour pour Kate se rend compte qu'il a échoué, car en la perdant il a perdu en même temps sa possibilité d'être heureux :

Nous aurions pu sauver le monde et nous aurions pu sauver le monde en un clin d'œil... mais nous ne l'avons pas fait, enfin je ne l'ai pas fait, et l'amour n'a pas triomphé, j'ai trahi l'amour et souvent quand je n'arrive plus à dormir c'est-à-dire à peu près toutes les nuits je réentends dans ma pauvre tête le message de son répondeur, "Hello this is Kate leave me a message », et sa voix était si fraîche, c'était comme plonger sous une cascade à la fin d'une poussiéreuse après-midi d'été, on se sentait aussitôt lavé de toute souillure, de toute déréliction et de tout mal. (100)

Il prend alors conscience qu'il aurait pu être heureux avec Camille : « Aurais-je pu vivre pendant des années seul avec Camille, dans cette maison isolée au milieu des bois, et être heureux ? Oui, je savais que oui » (311-312). Son mal-être est sans issue, sans illusions, total et a priori définitif. Peu de perspectives donc. Il reste la chimie qui aide à supporter, à placer à l'horizon un leurre ayant la forme du bonheur et vers lequel on marcherait comme vers une chimère. Le narrateur, comme Houellebecq dans son interview, reprend la fameuse phrase de Schopenhauer : «La possibilité du bonheur devait subsister ne fut-ce qu'à titre d'appât» (181). Le petit comprimé «fournit une nouvelle interprétation de la vie - moins riche, plus artificielle, et empreinte d'une certaine rigidité. Il ne donne aucune forme de bonheur, ni même de réel soulagement, son action est d'un autre ordre : transformer la vie en succession de formalités » (346).

Si Houellebecq dans ses romans précédents a construit un monde désabusé et désillusionné où il s'acharne à cerner différentes formes de bonheur incrustées dans des doctrines 
philosophiques, dans Sérotonine, il présente une vision cynique et pessimiste d'un univers où la possibilité de la vie en couple est dépassée, où l'avenir de la société humaine est mis en danger, où l'authenticité des civilisations et les valeurs de la culture contemporaine sont de plus en plus décadentes.

\section{Bibliographie}

Bruckner, Pascal. L'euphorie perpétuelle, essai sur le devoir du bonheur. Paris : Grasset, 2000.

Comte, Auguste. Cours de philosophie positive. Introduction et commentaires Florence Khodoss. Collection dirigée par Laurence Hansen-Løve. Edition numérique : Pierre Hidalgo La Gaya Scienza, 2012.

---. Correspondance générale et confessions Tome III avril 1845-avril 1846. Paris : La Haye et Mouton, 1975.

Debord, Guy. La société du spectacle. $3^{\mathrm{e}}$ édition. Paris : Gallimard, 1999.

Houellebecq, Michel. L'extension du domaine de la lutte. Paris : J'ai lu, 1994.

---. Les particules élémentaires. Paris : J'ai lu, 1998.

---. Interventions. Paris : Flammarion, 1998.

---. La possibilité d'une île. Paris : Fayard, 2005.

--- . Rester vivant. Paris : Librio, 2008.

--- . Sérotonine. Paris : Flammarion, 2019.

--- Interview. Festival de littérature de Louisiane, Musée d'art moderne Louisiana. Humlebæk : Danemark. Août 2019. https://www.youtube.com/watch?v=Rec-Ex9EJz0

Jérôme, David. «Auguste Comte toi-même !». L'unité de l'œuvre de Michel Houellebecq. Dir. Sabine Van Wesemael et Bruno Viard. Paris : Garnier, 2013. 137-148.

Lasch, Christopher. La culture du narcissisme. Paris : Champs-Flammarion, 2006.

Lebrun, Marie. Des nouvelles de Michel Houellebecq. http://marielebrun.unblog.fr/2009/10/08/des-nouvelles-de-michel-houellebecq/

Lipovetsky, Gilles. L'ère du vide, essais sur l'individualisme contemporain. Paris : Gallimard, 1983.

---. Les temps hypermodernes. Paris : Grasset, 2004.

Pommier, Gérard. «Attention bonheur !». Psychanalyse 1.1 (2004). 85-100. 
Rabosseau, Sandrine. «Michel Houellebecq ou le renouveau du roman expérimental ». Michel Houellebecq sous la loupe. Dir. Murielle Lucie Clément et Sabine van Wesemael, Amsterdam : Rodopi, 2007. 43-51.

Schopenhauer, Arthur. Le monde comme volonté et comme représentation. Paris : PUF, 1966.

Sonna, Birgit. «Conversation avec Michel Houellebecq sur le bonheur, l'échec de l'amour et la petite différence ». Texte Birgit Sonna. Photo Elias Hassos.

http://www.houellebecq.info/revuefile/33_Epart.PDF

Viard, Bruno. Houellebecq au laser : la faute à mai 68. Paris : Ovadia, 2008.

\section{Notes}

${ }^{1}$ A ce sujet voir: Auguste Comte Cours de philosophie positive.

L'état théologique ou féodal correspond au Moyen Âge et à l'ancien régime. Les relations sociales y sont analysées comme le résultat de l'idée surnaturelle de droit divin. Il est caractérisé par la croyance en des agents doués de volonté, qui expliquent tous les phénomènes frappants de la nature.

L'état métaphysique ou abstrait désigne le siècle des Lumières et notamment les encyclopédistes. Plus qu'un état, c'est le passage même de l'esprit théologique à l'esprit positif. Il peut selon les exemples paraître plus proche de l'esprit positif par la recherche de la rationalité ou plus proche de l'esprit théologique par le peu d'attention portée à l'observation des faits.

L'état scientifique ou positif : c'est l'état normal de l'intelligence à l'âge adulte. Cet état est définitif parce qu'il est conforme à notre condition et convient à nos besoins en même temps qu'à nos capacités.

${ }^{2}$ Le développement de l'individuation au vingtième siècle entraîne de graves conséquences pour l'être humain. Dans ce contexte, l'historien Christopher Lasch, dans La culture du narcissisme, discerne une sorte de performance de la vie quotidienne à travers un moi-acteur marqué par une emprise croissante de la conscience de soi. Il décrit une culture où l'individu s'examine, anxieux, à la recherche de signes de défaillances ou au contraire de bonne santé. Dans la postface de ce même essai, Lasch revient sur les effets sur la personnalité de ce qu'il nomme « la société du spectacle », selon l'expression de Guy Debord, en ces termes : «Les individus réagissaient les uns aux autres comme si leurs actions étaient enregistrées et simultanément transmises à un public invisible ou stockées pour une analyse ultérieure » (296). Le moi-acteur est sans cesse surveillé par les proches et les étrangers : «nous cherchons à nous rassurer sur notre pouvoir de captiver ou d'impressionner les autres, tout en demeurant à l'affût des imperfections qui pourraient nuire à l'apparence que nous voulons donner» (129). La critique de soi finit par étouffer toute spontanéité. Gilles Lipovetsky dans L'ère du vide, reprenant la pensée de Lasch, confirme : "c'est à un détachement émotionnel qu'aspireraient de plus en plus les individus, en raison des risques d'instabilité que connaissent de nos jours les relations personnelles » (109). L'être occidental moderne fait l'expérience de son moi solitaire, subit la séparation ontologique et n'est plus capable d'assumer la communication avec les autres, vit une perte des relations personnelles et manque de communion avec autrui. 